\title{
Antagonism between Staphylococcus epidermidis and Propionibacterium acnes and its genomic basis
}

Gitte J. M. Christensen ${ }^{1}$, Christian F. P. Scholz ${ }^{1}$, Jan Enghild², Holger Rohde ${ }^{3}$, Mogens Kilian ${ }^{1}$, Andrea Thürmer ${ }^{4}$, Elzbieta Brzuszkiewicz ${ }^{4}$, Hans B. Lomholt ${ }^{1}$ and Holger Brüggemann ${ }^{1 *}$

\begin{abstract}
Background: Propionibacterium acnes and Staphylococcus epidermidis live in close proximity on human skin, and both bacterial species can be isolated from normal and acne vulgaris-affected skin sites. The antagonistic interactions between the two species are poorly understood, as well as the potential significance of bacterial interferences for the skin microbiota. Here, we performed simultaneous antagonism assays to detect inhibitory activities between multiple isolates of the two species. Selected strains were sequenced to identify the genomic basis of their antimicrobial phenotypes.
\end{abstract}

Results: First, we screened 77 P. acnes strains isolated from healthy and acne-affected skin, and representing all known phylogenetic clades (I, II, and III), for their antimicrobial activities against $12 \mathrm{~S}$. epidermidis isolates. One particular phylogroup (I-2) exhibited a higher antimicrobial activity than other $P$. acnes phylogroups. All genomes of type I-2 strains carry an island encoding the biosynthesis of a thiopeptide with possible antimicrobial activity against $S$. epidermidis.

Second, 20 S. epidermidis isolates were examined for inhibitory activity against 25 P. acnes strains. The majority of $S$. epidermidis strains were able to inhibit $P$. acnes. Genomes of $S$. epidermidis strains with strong, medium and no inhibitory activities against $P$. acnes were sequenced. Genome comparison underlined the diversity of $S$. epidermidis and detected multiple clade- or strain-specific mobile genetic elements encoding a variety of functions important in antibiotic and stress resistance, biofilm formation and interbacterial competition, including bacteriocins such as epidermin. One isolate with an extraordinary antimicrobial activity against $P$. acnes harbors a functional ESAT-6 secretion system that might be involved in the antimicrobial activity against P. acnes via the secretion of polymorphic toxins.

Conclusions: Taken together, our study suggests that interspecies interactions could potentially jeopardize balances in the skin microbiota. In particular, S. epidermidis strains possess an arsenal of different mechanisms to inhibit $P$. acnes. However, if such interactions are relevant in skin disorders such as acne vulgaris remains questionable, since no difference in the antimicrobial activity against, or the sensitivity towards $\boldsymbol{S}$. epidermidis could be detected between health- and acne-associated strains of $P$. acnes.

Keywords: Staphylococcus epidermidis, Propionibacterium acnes, Skin microbiota, Bacterial antagonism, Antimicrobial substance, Bacteriocin, epidermin, ESAT-6 secretion system, Polymorphic toxin

\footnotetext{
* Correspondence: brueggemann@microbiology.au.dk

${ }^{1}$ Department of Biomedicine, Aarhus University, Aarhus, Denmark

Full list of author information is available at the end of the article
} 


\section{Background}

The microbiota of human skin is unique and complex, and is composed of a mixture of different groups of microorganisms: anaerobic, aerotolerant bacteria, such as propionibacteria; facultative anaerobic bacteria, such as staphylococci; fungi, such as Malassezia spp., and bacteriophages and viruses.[1, 2] According to both culture-based studies and metagenomic investigations, propionibacteria dominate in sebaceous sites and staphylococci and corynebacteria preferentially colonize moist areas [1-3]. However, different genera and species live in close proximity, in particular the two predominant skin species Staphylococcus epidermidis and Propionibacterium acnes. S. epidermidis colonizes various areas of the skin and is considered to be a skin commensal, but it can act as an opportunistic pathogen when it breaches the skin surface and enters the bloodstream $[4,5]$. P. acnes resides mainly in the pilosebaceous skin follicles; despite being a commensal with potential health-beneficial effects [6], evidence exists that the bacterium can also be an opportunistic pathogen, e.g., in postoperative infections $[7,8]$. The interaction between $S$. epidermidis and $P$. acnes and its relevance for skin health and disease of the host is largely unknown. This microbial interplay, for instance mediated through molecules involved in intercellular competition or communication, may have an impact on the fine balance of the skin ecosystem. A disturbed balance (dysbiosis) can impact skin health and might initiate or support the events that lead to skin disorders [9]. One of such disorders is acne vulgaris, a multifactorial disease of pilosebaceous units of the skin that affects adolescents [10]. Microorganisms have been associated with this disease, in particular $P$. acnes, and it has been reported that $S$. epidermidis and $P$. acnes can be isolated together from acne-affected sebaceous follicles [11].

Both species, $P$. acnes and $S$. epidermidis, are multiphyletic; $P$. acnes exhibits very limited strain- and cluster-level variation, whereas $S$. epidermidis strains and clades are more heterogeneous [12, 13]. Phylogenetic analyses can separate the $P$. acnes population into the clades I, II and III, which can be further subdivided into subclades and clonal complexes [12, 14-16]. Recently, the potential importance of $P$. acnes strain variation has been highlighted: certain lineages of $P$. acnes, in particular those of the I-1a cluster, are associated with acne, while other lineages are associated with healthy skin or deep tissue infections $[12,16,17]$.

Here, we investigated the antagonistic interactions between $P$. acnes and $S$. epidermidis, including strains of both species that have been isolated from healthy and acne-affected skin. Our study revealed various levels of antagonism between S. epidermidis and P. acnes and highlighted the importance of strain-level variation.
Genome sequencing of inhibitory S. epidermidis strains revealed an arsenal of fitness and competition functions encoded in the accessory genome. One potent strain possessed the epidermin biosynthesis cluster, and another strain harbours a functional type VII secretion system that might confer antimicrobial activity.

\section{Results}

Selection of bacterial strains and principal methodology Bacterial isolates, 77 and 24 strains for P. acnes and $S$. epidermidis, respectively, were previously obtained by swab sampling from human skin of acne patients and healthy individuals [12]. Some additional strains were obtained from the CCUG strain collection (Additional file 1 and Additional file 2). All P. acnes strains were phylogenetically analysed by multi-locus-sequence typing (MLST), using the scheme of Lomholt and Kilian [12]. The P. acnes isolates covered all major clades (I, II, III) and all sequence types assigned by Lomholt and Kilian [12].

Simultaneous antagonism assays were carried out. In the first set of experiments, the antimicrobial activity of $P$. acnes against $S$. epidermidis was investigated: strains of $S$. epidermidis were suspended on the agar surface, thus representing the indicator strains, and $P$. acnes was added as stab culture. In the second set of experiments, in order to assess the antimicrobial activity of S. epidermidis, $P$. acnes isolates were used as indicator strains in a similar set-up.

\section{$P$. acnes strains from the phylogroup $1-2$ displayed increased antimicrobial activity against $S$. epidermidis}

A total of $77 \mathrm{P}$. acnes strains were screened for their antimicrobial activity against $12 \mathrm{~S}$. epidermidis strains. A positive inhibitory effect was defined as a zone of inhibition around the $P$. acnes stab culture of at least $2 \mathrm{~mm}$. The $P$. acnes strains displayed varying degrees of antimicrobial activity against $S$. epidermidis. Among the $P$. acnes strains are potent inhibitors of $S$. epidermidis, such as strains 2.3.A1 (I-2, ST35) and 27.1.A1 (I-2, ST38) that inhibited 9 out of $12 \mathrm{~S}$. epidermidis strains. Other strains had little inhibitory effect, such as strains 5.1.R1 (II, ST52), CCUG33951 (II, 48) and CCUG50480 (I-1a, ST6) that inhibited only 1 out of $12 \mathrm{~S}$. epidermidis strains (Additional file 3).

One phylogroup, I-2, represented by 14 strains, displayed a significantly higher frequency of antimicrobial activity against $S$. epidermidis than the other phylogroups (I-1a, I-1b, III and II). P. acnes I-2 strains inhibited on average $61 \%$ of the tested $S$. epidermidis strains, whereas strains of the other $P$. acnes types inhibited on average only $29 \%$ of the tested S. epidermidis strains (Table 1). The reason for the broader antimicrobial activity of I-2 strains, i.e., inhibiting a higher 
Table 1 Antimicrobial activity of different phylotypes of $P$. acnes against $12 \mathrm{~S}$. epidermidis strains

\begin{tabular}{ll}
\hline Phylogroup & Antimicrobial activity in $\%^{\mathrm{a}}$ \\
\hline I-1a (43 strains) & $32 \%$ \\
I-1b (4 strains) & $42 \%$ \\
I-2 (14 strains) & $61 \%$ \\
III (2 strains) & $21 \%$ \\
II (14 strains) & $20 \%$ \\
\hline
\end{tabular}

a explanatory example: $P$. acnes $1-1$ a strains inhibited in average $32 \%$ of the tested S. epidermidis strains, whereas I-2 strains inhibited in average $61 \%$ of the tested S. epidermidis strains

proportion of the tested S. epidermidis strains, is likely to be found in the genomic regions that are specific for I-2 strains. On the accessory genome of type I-2 strains a genomic island is located that encodes a thiopeptide similar or identical to berninamycin A of Streptomyces bernensis $[18,19]$.

No difference in prevalence and intensity was noted in the antimicrobial activity of $P$. acnes strains isolated from normal and acne-affected skin (Table 2). Among the I-1a strains, isolates from acne patients were not distinct in their anti-S. epidermidis activity from isolates obtained from healthy skin. This suggests that a particular antimicrobial activity of $P$. acnes against $S$. epidermidis does not play a role in the acne-associated microbiota.

\section{Strain-dependent antimicrobial activity of S. epidermidis against $P$. acnes}

The results of the antagonism assay were then analysed from the perspective of the antimicrobial activity of $S$. epidermidis. Eleven out of 20 S. epidermidis strains displayed varying degrees of antimicrobial activity against 25 P. acnes strains (Table 3). Among the S. epidermidis strains with an elevated antimicrobial activity, differences in inhibition zone diameter and appearance were observed, indicating that the antimicrobial substances were of different nature (Fig. 1). Most of the S. epidermidis strains displayed small zones of inhibition (2- $4 \mathrm{~mm}$ ) against $P$. acnes and some strains produced opaque zones of inhibition. Interestingly, one strain, FS1, produced very large inhibitory zones $(>10 \mathrm{~mm})$, but was not active against all $P$. acnes strains tested. Another strain,

Table 2 Origin of $P$. acnes strains and their antimicrobial activity against $\mathrm{S}$. epidermidis

\begin{tabular}{ll}
\hline Skin status & Antimicrobial activity in \% \\
\hline healthy skin (22 strains) & $38 \%$ \\
light acne (17 strains) & $34 \%$ \\
moderate acne (11 strains) & $39 \%$ \\
severe acne (6 strains) & $29 \%$ \\
\hline
\end{tabular}

Table 3 Antimicrobial activity of 20 S. epidermidis strains against 25 P. acnes strains

\begin{tabular}{lll}
\hline S. epidermidis strain & Isolated from & $\begin{array}{l}\text { Antimicrobial } \\
\text { activity in } \%^{a}\end{array}$ \\
\hline 14.1.R1 & upper back, light acne & 100 \\
AU40 & healthy alar crease & 88 \\
AU21 & healthy nares & 72 \\
FS1 & face, light acne & 68 \\
GS3 & face, moderate acne & 64 \\
AU23 & healthy nares & 64 \\
AU48 & healthy nares & 28 \\
AU39 & healthy alar crease & 12 \\
AU60 & healthy nares & 8 \\
AS1 & alar crease, light acne & 8 \\
AU36 & healthy nares & 4 \\
AU10, AU16, AU24, & diverse, see Additional & 0 \\
AU35, AU44, AU53, & file 2 & \\
AU73, AU81, IS2 & &
\end{tabular}

aisted from most potent to least potent strain, e.g., $100 \%$ refers to an antimicrobial activity of $S$. epidermidis against all $25 P$. acnes strains

14.1.R1, was able to inhibit all P. acnes strains, but produced small zones of inhibition (2-5 mm). No difference in prevalence and intensity was noted in the antimicrobial activity of $S$. epidermidis strains isolated from normal and acne-affected skin, respectively (Table 3). Likewise, the origin of $P$. acnes strains did not determine their susceptibility to the antimicrobial activity of $S$. epidermidis, since strains isolated from acne lesions and healthy skin, respectively, were not significantly different in their susceptibility patterns towards $S$. epidermidis (Additional file 4).

\section{Antimicrobial activities of S. epidermidis strains 14.1.R1 and FS1}

To further characterize the observed broad antimicrobial activity of $S$. epidermidis strain 14.1.R1, the screening was extended with 29 additional $P$. acnes strains representing all phylogroups. All additional P. acnes strains were also inhibited by this $S$. epidermidis strain (Additional file 5). To test whether the antimicrobial activity of $S$. epidermidis strain 14.1.R1 was P. acnes-specific, other Gram-positive and Gram-negative bacteria were also screened. S. epidermidis strain 14.1.R1 did not have the ability to inhibit any of the other bacterial species tested, except for a Propionibacterium granulosum strain. This shows that the antimicrobial activity of strain 14.1.R1 is P. acnes-specific, and might be active against closely related propionibacteria as well, but not against species of other genera.

Strain FS1 produced the largest inhibition zones of all tested S. epidermidis strains. It was particularly active against $P$. acnes type II and III strains (inhibition 


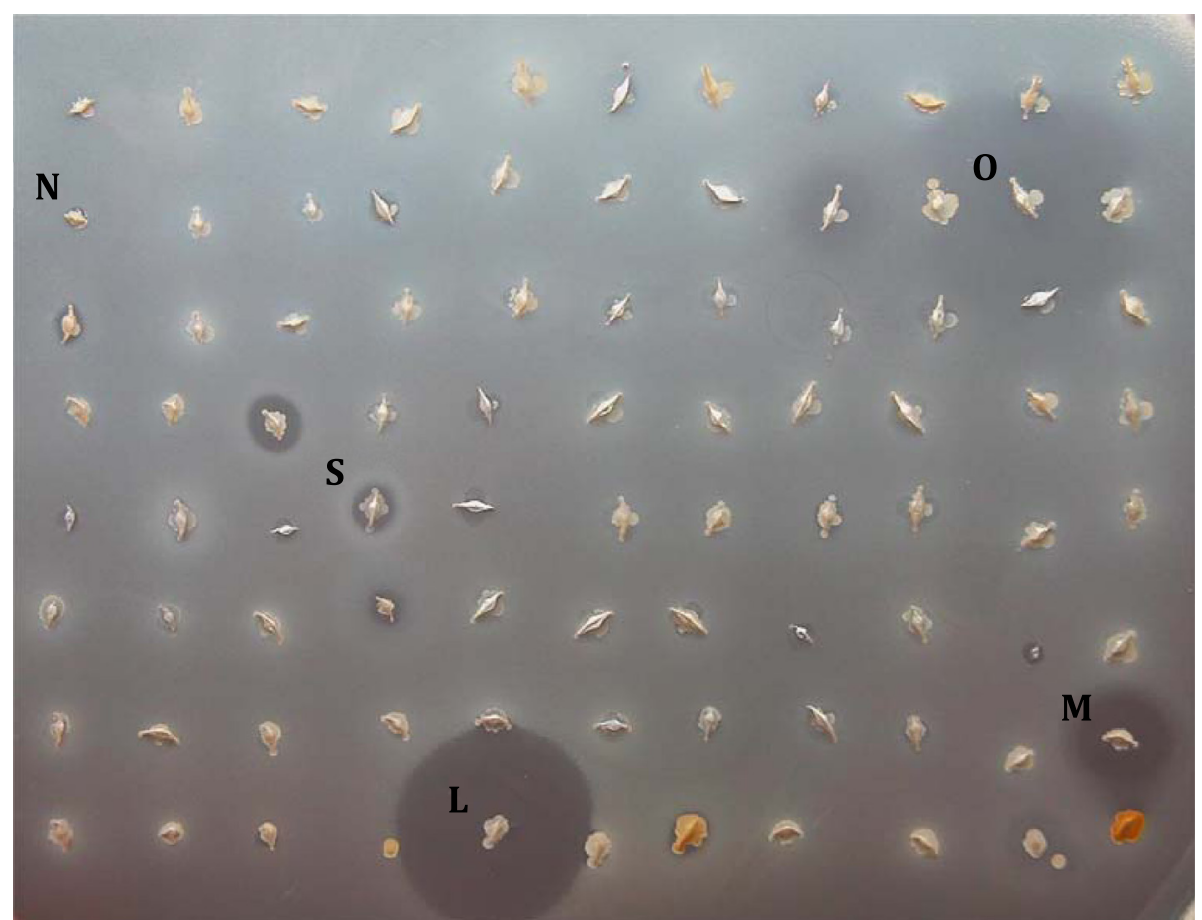

Fig. 1 Simultaneous antagonism assay of S. epidermidis against $P$. acnes. A representative result of a simultaneous antagonism assay is shown. A large TS agar plate was floated with a suspension of a $P$. acnes indicator strain, and strains of $S$. epidermidis were point-inoculated on the agar surface. Growth inhibition around several of the $S$. epidermidis colonies is apparent. Diameters of the depicted inhibitory zones ranged from $3 \mathrm{~mm}$ to $20 \mathrm{~mm}$. Arrows point to the individual zones of inhibition, i.e., opaque $(\mathrm{O})$, small $(\mathrm{S})$, medium $(\mathrm{M})$, large $(\mathrm{L})$ zones, or no inhibition zone $(\mathrm{N})$

zone $>10 \mathrm{~mm})$. A diffuse, smaller inhibition zone against most $P$. acnes type I strains was observed (data not shown).

\section{Comparative genomics of S. epidermidis highlights core genome diversity}

In order to investigate the genetic basis for the antimicrobial activity of $S$. epidermidis against $P$. acnes, strains with broad (14.1.R1), medium (FS1, AU21, AU23) and no (AU24) inhibitory activities were selected and genome sequenced. One strain (AU23) was sequenced with high quality by PacBio technology and the three other isolates (FS1, AU21, AU24) were Illumina-sequenced. The genome sequence of the strain 14.1.R1 was taken from GenBank (accession number AGUC00000000; 2,555,725 bp in 131 contigs). The genome of strain FS1 has a similar size with 2,551,451 bp (in 91 contigs). The genomes of strains AU21 $(2,421,814 \mathrm{bp}$ in 72 contigs) and AU24 $(2,429,243$ bp in 61 contigs) are considerably smaller compared to 14.1.R1 and FS1, on average $130 \mathrm{~kb}$. The genome size of strain AU23 is 2,487,973 bp (in 5 contigs); one contig of $42 \mathrm{~kb}$ represents a plasmid. To assess the phylogenetic distance of the five isolates and to relate them to other $S$. epidermidis strains, a phylogenetic tree was constructed based on the core genome of $112 \mathrm{~S}$. epidermidis genome sequences (Fig. 2). The size of the core genome is approximately 1.7 Mb, which is in concordance with previous findings [13]. The tree shows that the $S$. epidermidis population is divided into two main clades, with a large heterogeneity within each clade. The core genomes of AU21 and AU23 are almost identical, and they group together in the same clade as strain AU24. Strain FS1 belongs to the same clade, but is located in an outlier cluster, indicating increased phylogenetic distance to strains AU21, AU23 and AU24. The strain 14.1.R1 is phylogenetically most distinct and clusters in the second clade. Thus, antimicrobially active $S$. epidermidis strains are scattered in phylogenetically not closely related clades. This indicates that the inhibitory activity of $S$. epidermidis against $P$. acnes is not part of the core genome, but is rather encoded in the accessory genome.

The accessory genome encodes diverse fitness functions including factors involved in interbacterial competition In order to identify the genomic basis for the antimicrobial activity against $P$. acnes, we focussed on genomic regions in strains AU21, AU23, FS1 and 14.1.R1 that are absent in strain AU24, the isolate without inhibitory activity. Comparative genome analysis revealed that all 


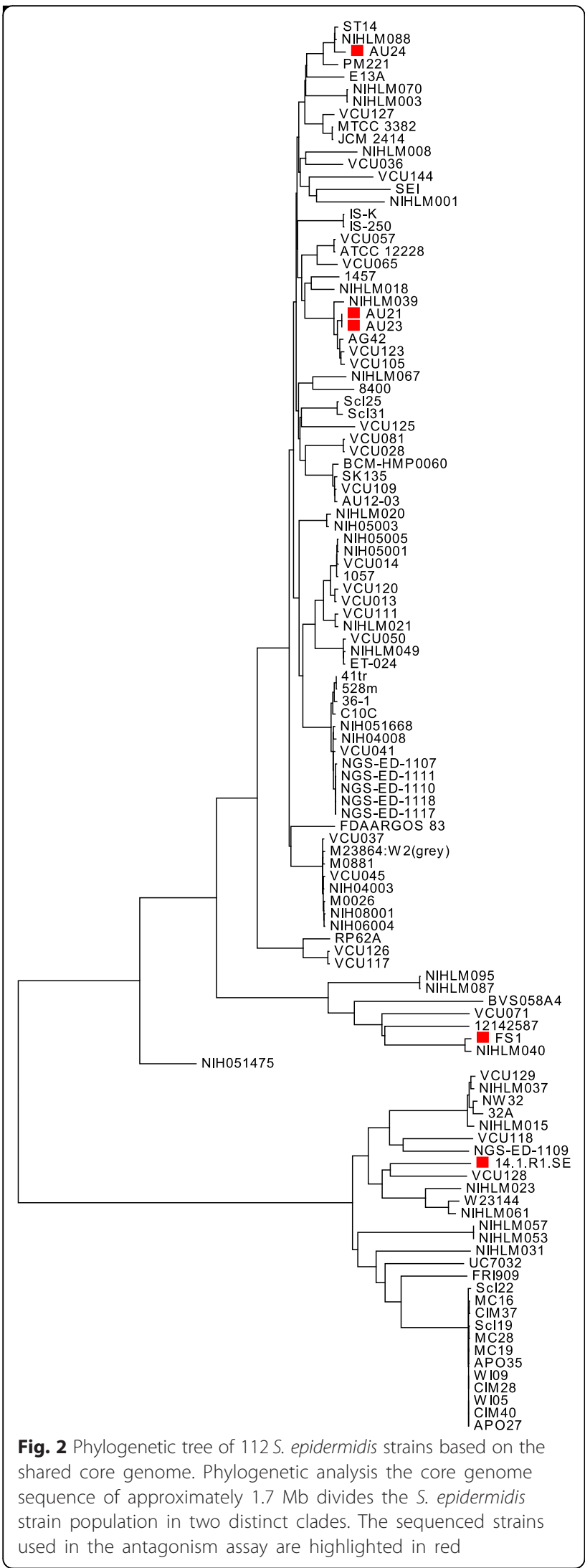

strains comprise several mobile genetic elements that are only rarely found in other sequenced S. epidermidis strains, and that confer a variety of fitness functions, including antibiotic and stress resistance, biofilm formation, interbacterial competition and, possibly, virulence. A comprehensive analysis was done for each strain:

\section{AU21 and AU23}

Strains AU21 and AU23 had similar moderate antimicrobial activities against $P$. acnes, and produced diffuse inhibitory zones. The accessory genomes of both strains are largely identical, underlining their close phylogenetic relationship. The accessory genome of strains AU21 and AU23 is distributed in six larger regions, that are absent in the chromosome of strain AU24 (Additional file 6A and Additional file 7A). A variety of functions can be found. One region (region 1 in Additional file 6A) is rarely found in any other sequenced S. epidermidis genome; it is a genomic resistance island of possible phage origin, encoding FusB that confers resistance to the antibiotic fusidic acid. This island has recently been described in S. epidermidis strain NTUH-3692 [20]. Another genomic island (region 3) encodes proteins conferring increased tolerance to divalent metal ions such as zinc, copper and cobalt. Region 5 encodes an arginine deiminase (ADI) system, catalyzing the conversion of arginine to ornithine via citrulline, thereby generating $\mathrm{NH}_{3}$ and ATP. The ADI system is used by several bacterial species to facilitate survival in acidic conditions [21]. At least two other islands (region 2 and 4) encode proteins related to the bacterial surface, such as teichoic acid modifying enzymes and the cell surface proteins SasG and SasC that are implicated in host interactions and biofilm accumulation [22]. The $42 \mathrm{~kb}$ plasmid (region 7) encodes among others several transport systems, a putative lysine (or arginine) biosynthesis gene cluster, and proteins protective against oxidative stress such as glutathione peroxidase/reductase (Additional file 7A).

The shared accessory genome of AU21 and AU23 encodes no known bacteriocin or any other previously identified antimicrobial system. We conclude that the inhibitory activity of these strains is conferred either by an unknown bacteriocin or bacteriocin-like substance, or by other means, such as the secretion of the fermentation product succinic acid, which has been shown to inhibit $P$. acnes [23]. In this regard, the ADI system found in AU21 and AU23 might increase self-protection of succinic acid-producing cells from the adverse effects of acidification.

\section{FS1}

Strain FS1 produced the largest zones of inhibition against most $P$. acnes strains. Its genome harbors 10 
genomic regions that are absent from strain AU24 and most other sequenced $S$. epidermidis strains, including the three examined strains AU21, AU23 and 14.1.R1 (Additional file 6B and Additional file 7B). Several regions encode phage-related proteins (regions 3, 4, 8 and partially 10 in Additional file 6B). Region 8 has similarity to $S$. aureus superantigen-encoding pathogenicity islands (SaPIs). These phage-related chromosomal islands are considered to be phage satellites or parasites [24]. Another island (region 6) represents the well-characterized biofilm operon icaADBC that encodes the polysaccharide intercellular adhesin (PIA) [25]. Two genomic regions are interesting with regards to antimicrobial activity. Region 1 represents a full or partial plasmid, as judged from the presence of genes encoding plasmid replication proteins and a toxin-antitoxin system conferring plasmid stabilisation (Additional file 7B). This $42 \mathrm{~kb}$ region harbors an operon encoding a bacteriocin, belonging to the lactococcin-972 superfamily (Pfam09683), an immunity protein as well as a putative bacteriocin export $\mathrm{ABC}$ transporter. The other genomic region encoding an antimicrobial substance is a $12 \mathrm{~kb}$ cluster located in region 10. It encodes EpiA, the precursor peptide for the well-characterized lantibiotic epidermin, as well as proteins involved in posttranslational modification of EpiA, including homologs of EpiBCDRP [26]. Epidermin has been shown to act on several Gram-positive bacteria, including $P$. acnes. Interestingly, epiP is frameshifted in the genome of FS1, resulting in a truncated protein. EpiP is the precursor-processing protease that cleaves the 52 -aa precursor peptide into the mature 22-aa bioactive peptide [27]. This raises doubts about the activity of the epidermin(-like) peptide in strain FS1. Biosynthesis of epidermin is encoded on a $54 \mathrm{~kb}$ plasmid in S. epidermidis strain Tü3298 [26]. Thus, it is likely that the $12 \mathrm{~kb}$ epiA containing contig (region 10) and the $42 \mathrm{~kb}$ plasmid contig (region 1) together constitute a $54 \mathrm{~kb}$ plasmid. In summary, the antimicrobial activity of strain FS1 likely derives from one or both identified bacteriocins.

\subsection{R1}

Next, we analysed the genome of S. epidermidis 14.1.R1, the strain with extraordinary antimicrobial activity against $P$. acnes. The genome comparison revealed eight large genomic islands, some of which are only found in strain 14.1.R1 (Fig. 3). Signatures of their horizontal acquisition can be detected in these clusters, such as an aberrant GC content and/or GC skew, and mobilityassociated functions including replication proteins and integrases (Fig. 3 and Additional file 7C). Region 1 encodes antibiotic resistance determinants (beta-lactamase) and a recombinase system similar to the Staphylococcal Cassette Chromosome mec (SCCmec). Region 2 encodes an alternative secretion system, the accessory Sec system (SecA2-SecY2) that is dedicated to the export of a family of glycosylated serine-rich repeat proteins [28]. Region 3 constitutes a prophage genome that is also present in Staphylococcus capitis. Regions 4 and 6 represent phage-related chromosomal islands with similarity to SaPIs [24]. The SaPI-like region 4 harbours the putative virulence gene $v a p E$ as previously noted in the literature [20]. Region 5 harbors a large gene $(4.6 \mathrm{~kb})$ encoding a biofilm-associated protein (Bap) that has been shown to be important in biofilm formation independent of the PIA/PNAG exopolysaccharide [29]. Region 7 is described below in more detail. Region 8 is composed of 7 different contigs varying between 3 and $14 \mathrm{~kb}$ in size. Some of these contigs appear to be plasmids. Most S. epidermidis strains contain plasmids; one to six plasmids are present in the strains that have been completely sequenced to date. Strain 14.1.R1 contains most likely several plasmids. For example, a $14 \mathrm{~kb}$ contig (AGUC01000118) has similarity to S. epidermidis plasmid SAP110A (NC_013383) that encodes antibiotic resistance functions.

\section{S. epidermidis strain 14.1.R1 harbors the ESAT-6 secretion system}

A $27 \mathrm{~kb}$ genomic region (region 7, located on contig AGUC01000069) of strain 14.1.R1 is of particular interest (Additional file 7C). This island is absent in most sequenced $S$. epidermidis strains, but a BLASTn analysis showed partial homology to $S$. aureus. The $27 \mathrm{~kb}$ region harbors 43 genes (Fig. 4); among them are seven genes (esx $A, e s a A, e s s A, e s a B, e s s B, e s s C$, and $e s x B$ ) that encode components of the 'early secretory antigenic $6 \mathrm{kDa}$ (ESAT-6) secretion system (ESS) and ESS-secreted substrates. To our knowledge, this Sec-independent secretion system has so far not been described in any other $S$. epidermidis strain. The ESS, with similarity to the type VII secretion of Mycobacterium tuberculosis, has been studied in S. aureus, where it was found to be involved in virulence via the secretion of various factors, including EsxA, EsxB, EsaC and EsaD [30-32]. Strikingly, EsxA was detected in this study in the bioactive supernatant of S. epidermidis 14.1.R1 by mass spectrometry (see below), indicating that the ESS is functional and might contribute to the special antimicrobial phenotype of this strain. Besides the $12 \mathrm{~kb}$ ESS locus, other genes within this $27 \mathrm{~kb}$ region might be functionally linked to the secretion system. The genes downstream of the ESS cluster encode several polymorphic toxins with nuclease domains and associated immunity proteins. It is hypothesized that such nuclease toxins can confer contactdependent growth inhibition (see discussion). 


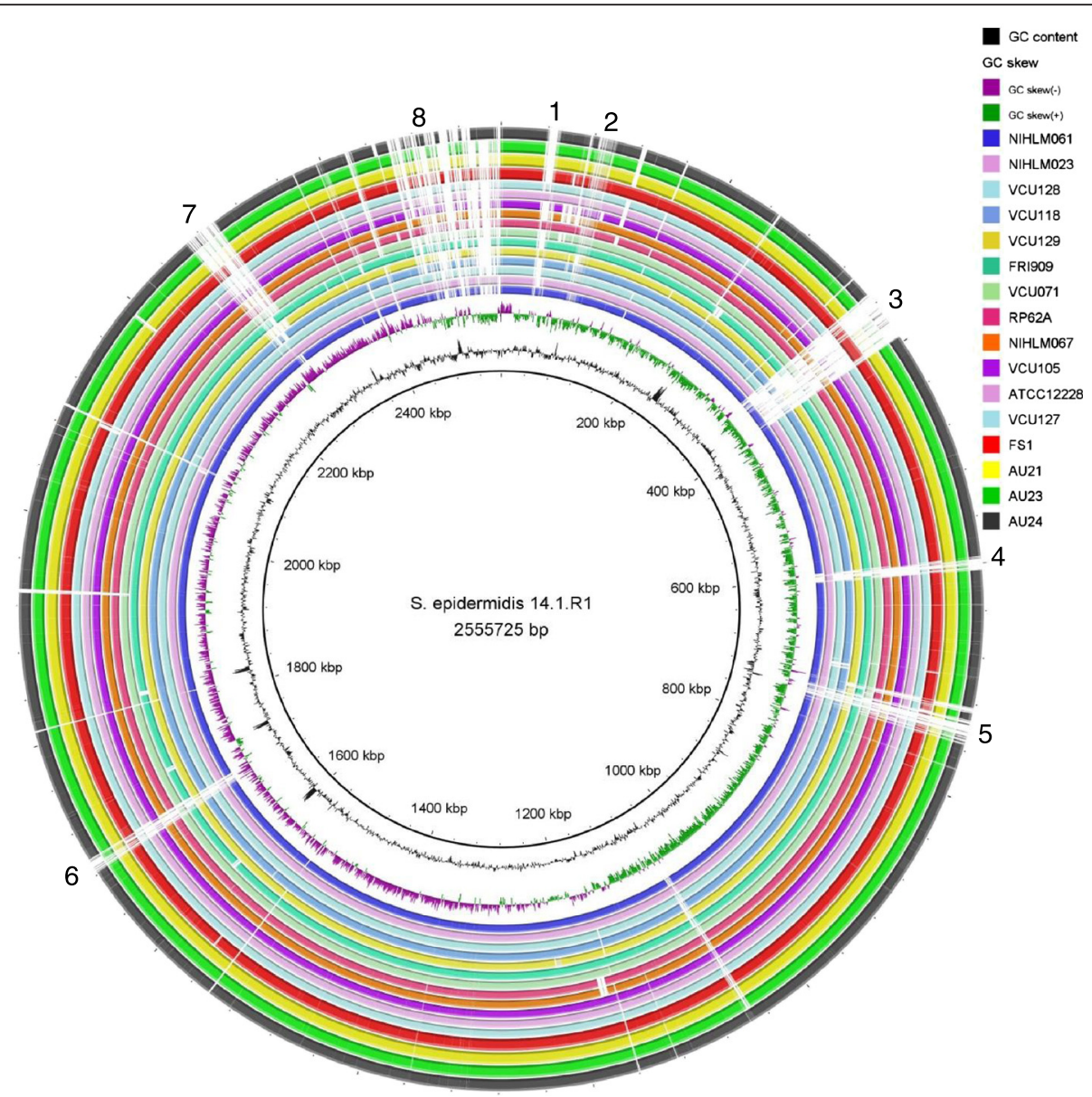

Fig. 3 Genome comparison of 17 S. epidermidis strains. Strain 14.1.R1 was used as the reference genome. The four newly sequenced genomes of strains FS1, AU23, AU21 and AU24 are represented by the four outermost rings. The other 13 genomes were selected based on their position in the phylogenetic core genome tree, which shows the clustering of strains VCU128, NIHLM023, NIHLM061, VCU118 and VCU129 with strain 14.1.R1 (Fig. 2). Regions of genomic variability (1-8) can be detected (see Additional file $7 C$ for their gene content). The BRIG program was used to generate the figure

\section{Partial purification and initial characterization of the} bioactive compound from S. epidermidis 14.1.R1

It was noted that the antimicrobial activity of strain 14.1.R1 against $P$. acnes could be obtained only when $S$. epidermidis was grown on agar plates; no significant antimicrobial activity could be detected when S. epidermidis was grown in liquid medium. Hence, a partial purification of the bioactive compound of strain 14.1.R1 was tried using agar plate-grown S. epidermidis. A concentrated cell-free supernatant (cCFS) extract was obtained from harvesting 45 agar plates. It was noted that bioactivity was rapidly lost upon dilution of the cCFS. Stability of the bioactivity was reduced when storing the cCFS at $4{ }^{\circ} \mathrm{C}$ in non-coated Eppendorf tubes, indicating

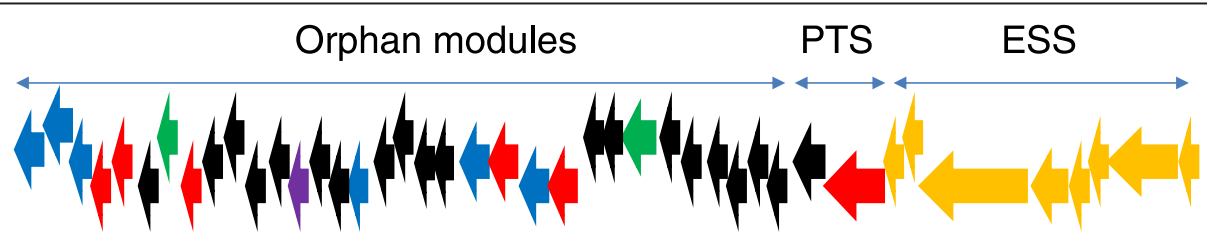

Fig. 4 Genomic locus in S. epidermidis 14.1.R1 encoding an ESAT-6 secretion system and multiple nuclease/immunity protein pairs. In orange, ESAT-6 secretion system (ESS) and secreted effectors (from right to left: esXA, esaA, essA, esaB, essB, essC, unknown effector, esxB), In red: proteins with DNA/RNA nuclease domains, possible toxin components; in green, SMI1/KNR4 family (SUKH-1) domain proteins, possible immunity components; lila, serine protease (NfeD-like protein); in blue, proteins with domains of unknown function (DUF600, DUF1851, DUF4467). PTS, polymorphic toxin system. See Additional file 7C for the genomic location and annotation 
non-specific adsorption of the bioactive substance to the vessel walls. Coating of the tubes with Emulphogene could prevent the time-dependent loss of activity. Exposure of the cCFS to proteinase $\mathrm{K}$ resulted in a complete loss of inhibitory activity, suggesting that the active substance is of proteinaceous nature. Antimicrobial activity was unaffected following exposure to low $\mathrm{pH}(\mathrm{pH} 2)$ for $2 \mathrm{~h}$ with subsequent neutralization. Heat treatment of the cCFS $\left(60{ }^{\circ} \mathrm{C}\right.$ for $\left.10 \mathrm{~min}\right)$ led to complete loss of inhibitory activity.

\section{Identification of proteins in the bioactive cCFS extract of S. epidermidis 14.1.R1}

In an attempt to identify the antimicrobial compound produced by $S$. epidermidis 14.1.R1, the cCFS containing the bioactivity against $P$. acnes was analysed by MS. The cCFS extract was separated in different concentrations by SDS-PAGE and visible bands were subject to MS analysis (Additional file 8). Two proteins, the phenol soluble modulin (PSM) $\beta 1$ (MW: $4639 \mathrm{Da}$ ) and PSM 32 (MW: $4644 \mathrm{Da}$ ) were identified in one band. Homologs of PSM $\beta 1$ and PSM $\beta 2$ were found in the genome of strain 14.1.R1 (Additional file 9). The non-cytolytic PSM $\beta$ proteins have been previously investigated; they are associated with promotion of biofilm structuring, maturation and detachment in vitro [33,34]. It is unlikely that the non-cytolytic PSM $\beta$ proteins are responsible for the antimicrobial activity of strain 14.1.R1, since these proteins are not unique to strain 14.1.R1, but present in many $S$. epidermidis strains, including the non-inhibiting strain AU24. Moreover, the PSM $\beta$ proteins are heat-resistant [35], but we showed that the bioactive protein of strain 14.1.R1 is heat-labile.

A second band of the SDS-PAGE gel was identified as EsxA (HMPREF9956_2246) (Additional file 10). This protein is encoded in the ESS island described above, and is a known effector protein secreted by the ESS. The function of this protein in S. epidermidis needs to be explored in the future.

Another protein band was identified as a protein similar to DNA-binding protein $\mathrm{HU}$ of $S$. aureus strain Mu50 (HMPREF9956_1196) (data not shown). This histone-like protein belongs to the nucleoid-associated proteins and contributes to chromosomal compaction and maintenance of negative supercoiling.

Taken together, these data indicate that S. epidermidis strain 14.1.R1 secretes putative virulence factors such as PSMs and EsxA. The fact that EsxA was detected indicates that the ESAT-6 secretion system is functional in S. epidermidis 14.1.R1.

\section{Discussion}

Interspecies competition is a common phenomenon among members of the (human) microbiota; it can be conferred by several means. Prevalent are secreted antimicrobial substances of the bacteriocin family that can kill competing bacteria with different mechanisms, for example by pore-forming, nuclease, or cell wall synthesisinterfering activities. In addition, primary or secondary metabolites also impact on the microenvironment of the producing cell; this can result in the inhibition of competing bacteria in the close vicinity.

This study examined the antimicrobial action of $P$. acnes against $S$. epidermidis and vice versa as determined by an in vitro simultaneous antagonism assay. In agreement with previous findings, bacterial antagonism between $P$. acnes and $S$. epidermidis is a common phenomenon $[23,36]$. Our study revealed a higher frequency of antimicrobial activity among $P$. acnes strains belonging to the I-2 phylogroup as compared with other phylogroups of $P$. acnes against $S$. epidermidis. A likely possibility is the presence and secretion of a bacteriocin or bacteriocin-like substance specific to phylogroup I-2. A genomic comparison previously identified some phylogroup I-2-specific genes $[18,19]$. Among these, a thiopeptide biosynthesis gene cluster located in a genomic island is present in all genomes of I-2 strains. Transcriptome analyses of $P$. acnes have highlighted the activity of this genomic island in the I-2 phylogroup of $P$. acnes [18]. Two thiopeptide precursor peptides are encoded in the $P$. acnes thiopeptide gene cluster; one precursor possesses the 16-aa structural peptide of berninamycin A from Streptomyces bernensis [37]. We therefore assume that the $P$. acnes thiopeptide has structural similarity or identity to berninamycin A. Thiopeptides such as berninamycins have been isolated from soil-derived bacteria of the Streptomyces genus and from marine sources, and are peptides with an antimicrobial potential that are potent inhibitors of protein synthesis in Grampositive bacteria [38, 39]. Thus, we hypothesize that a thiopeptide produced by $P$. acnes type I- 2 strains could be responsible for the more frequent antimicrobial activity against $S$. epidermidis. Future research needs to be done to confirm this hypothesis.

With regard to the antimicrobial activity of $S$. epidermidis against $P$. acnes, the current study revealed a strain-dependent pattern of antimicrobial activity among S. epidermidis. In agreement with the study of Holland et al. [36], we found no difference in the antimicrobial susceptibility among $P$. acnes isolates from healthy or acne-affected individuals. This might mean that susceptibility/resistance of $P$. acnes towards the antimicrobial activities of $S$. epidermidis is not important in the acne pathogenesis.

Our study showed a large variation of the antimicrobial activity among $S$. epidermidis strains against $P$. acnes. Among the inhibitory strains, the nature and size of inhibition zones varied, indicating that different 
antimicrobial mechanisms are at play. Comparative genome analyses revealed that there was no strong phylogenetic relatedness between inhibitory S. epidermidis strains. This indicates that the antimicrobial activity is not encoded on the core genome, but rather encoded on mobile genetic elements (MGEs) that are exchanged through horizontal gene transfer. Accessory traits, such as the ability to produce antimicrobial substances, have often been found to be encoded on MGEs in staphylococci [40-42].

Four strains with no, medium or broad antimicrobial activities were sequenced in order to determine the genomic basis of their respective phenotypes. Based on the sequence data different possible mechanisms conferring antimicrobial activity were identified.

1. For strains AU21 and AU23 no known bacteriocin gene was found to be encoded in the accessory genome. It is possible that these strains employ an unknown bacteriocin or an entirely novel mechanism. Alternatively, the production of metabolic products could have anti-P. acnes effects. A candidate is succinic acid that has been identified to be produced by $S$. epidermidis during fermentation [23]. This acid is able to kill $P$. acnes by lowering its intracellular $\mathrm{pH}$. The producer strain needs to develop a self-protective mechanism against acidification. Interestingly, AU21 and AU23 carry an arginine deiminase (ADI) system that can confer such protection via the production of $\mathrm{NH}_{4}$. The ADI system has been shown to be crucial for resistance to acid stress in certain S. epidermidis strains [43]. The involvement of a metabolite such as succinic acid could also explain the diffuse nature of the inhibitory zone, which might result from incomplete lysis of $P$. acnes.

2. The strain FS1 produced large inhibition zones. Its genome contains two gene clusters encoding bactericoins; both clusters are likely to be encoded on a plasmid of $54 \mathrm{~kb}$. One bacteriocin is of the lactococcin-972 superfamily and has not previously been studied to our knowledge. It is 130 aa in size and harbors an N-terminal signal peptide of 25 aa, indicative of Sec-dependent export. A putative immunity protein with transmembrane helices and a transporter are encoded immediately downstream. The second bacteriocin is a lantibiotic. The precursor peptide is identical to epidermin, a well-characterized lantibiotic of $S$. epidermidis [26]. Epidermin and the closely related gallidermin act on the cytoplasmic membrane of Grampositive bacteria. Gallidermin was successfully tested in a topical formulation on rat skin showing antibacterial activity against $P$. acnes and $S$. aureus [44]. Due to the detected frameshift mutation of epiP in strain FS1, it has to be investigated in the future if the epidermin precursor peptide is not cleaved or processed differently in strain FS1.

3. Our study further revealed that the S. epidermidis strain 14.1.R1 had a broad inhibitory activity; it was the only tested $S$. epidermidis strain that inhibited all tested $P$. acnes strains. By a whole genome comparison approach, we searched for the underlying gene(s) responsible for the exceptional phenotype of S. epidermidis 14.1.R1. One genomic island was of special interest, the island was neither found in strain AU24, nor in most other genomes of sequenced S. epidermidis strains. Encoded on this island is a specialized ESAT-6 secretion system (ESS), which is homologous to the type VII secretion system of $M$. tuberculosis [30, 45]. This system in mycobacteria is required for full virulence as it has roles in the survival of mycobacteria in macrophages, granuloma formation, induction of apoptosis and autophagy, phagosomal rupture, and host cell lysis [30, 45-48]. The ESS has also been described in other Gram-positive bacteria, including S. aureus [31, 32, 45], but it has not yet been described in S. epidermidis. ESS-secreted effectors, belonging to the WXG100 family of proteins, whereof ESAT-6 and CFP-10 from $M$. tuberculosis are the founding members, have been identified in S. aureus, designated EsxA and EsxB for ESAT-6 extracellular protein A and B, respectively [31, 49-52]. No clear biological function has been attributed to the ESS effector proteins in S. aureus to date, but mutants that failed to secrete EsxA and EsxB displayed defects in abscess formation in mice, suggesting that these proteins are important during staphylococcal disease [31]. A recent study showed that EsxA interferes with host cell apoptotic pathways and, together with EsxB, mediates the release of intracellular $S$. aureus from the host cell [52]. The ESS is most likely functional in strain $S$. epidermidis 14.1.R1 as we could detect EsxA in the cell-free supernatant. However, it is unlikely that EsxA is responsible for the exceptional antimicrobial activity of S. epidermidis 14.1.R1 against P. acnes, since EsxA homologs have not been described as antimicrobial but rather as host cell-interacting factors involved in persistence and spread of the bacterium in the host. $[31,51,52]$ The genomic island encoding the ESS has another interesting feature that could be linked to, or responsible for, the antimicrobial activity of strain 14.1.R1. Bioinformatics analysis revealed that the island contains other possible ESS effectors: The protein encoded downstream of 
es $x B$ has an $\mathrm{N}$-terminal LXG domain, a possible recognition site for a type VII secretion system [53]; its $\mathrm{C}$-terminus encodes a predicted ribonuclease domain, which is a toxin component of the YeeFYezG toxin-antitoxin module. In fact, this protein represents a polymorphic toxin; such toxins have recently been discovered to mediate interbacterial competition [53-55]. Moreover, many of the 34 genes downstream of ESS encode other toxins and immunity proteins of the SUKH superfamily. These toxin-immunity protein pairs are orphan modules, encoding alternate toxin domains and their cognate immunity proteins; such orphan modules have been found in many bacteria [53-56]. The toxin domains include DNA/RNA non-specific endonuclease (pfam13930); nuclease of the HNH/ENDO VII superfamily (pfam14414); toxin 43 superfamily (pfam15604) and serine protease (NfeD-like protein). Some polymorphic toxin systems are involved in contact-dependent growth inhibition (CDI) $[56,57]$. CDI has been reported in proteobacteria; direct contact with the target cell is required to deliver nuclease toxins into target cells. Taken together, we hypothesize that a type VII-like secretion system in S. epidermidis 14.1.R1 is able to deliver polymorphic toxins directly onto or into target bacteria resulting in CDI. This could be a possible explanation of the broad antimicrobial phenotype of strain 14.1.R1 against $P$. acnes, with the rather small inhibitory zones produced by 14.1.R1. It would also explain why we were unable to purify the antimicrobial substance and why the anti-P. acnes activity was only observed when S. epidermidis 14.1.R1 was grown on agar plates.

Further studies are required to assess the in vivo relevance of the anti- $P$. acnes activity exerted by many $S$. epidermidis strains. The method used in this study is an in vitro antagonism assay. The detectable inhibitory activity is dependent on growth conditions such as the medium composition and the applied incubation conditions (e.g., oxygen levels). Therefore, it is not clear to what degree the inhibition phenomena obtained on agar medium reflect the in vivo settings on human skin. It further needs to be clarified if the antimicrobial activity results in a competitive advantage of the producing strain in vivo and how this impacts on the skin ecosystem.

\section{Conclusions}

This study examined antagonistic interactions of two prominent members of the skin microbiota, P. acnes and S. epidermidis. The study showed a variety of clade- and even strain-specific inhibitory activities. Genome sequencing of selected S. epidermidis isolates highlighted the heterogeneity of this species, and the presence of multiple genomic islands. Accessory genome analyses identified several possible inhibitory mechanisms, including the secretion of acidic fermentation products and the respective self-protection via ADI, the production of bacteriocins such as epidermin, and the employment of a type VII-like secretion system (ESS) that might deliver polymorphic toxins on or into the target cell. The functionality of this system in S. epidermidis is likely, as judged from the identification of an ESS effector in the culture supernatant.

As a possible application, it could be envisaged that the diverse anti- $P$. acnes activity of $S$. epidermidis could be exploited, for example as a probiotic treatment approach against acne vulgaris or other $P$. acnes-associated diseases.

\section{Methods}

\section{Bacterial strains}

S. epidermidis and $P$. acnes strains originating from healthy and acne-affected individuals were obtained by skin swabs using cotton sticks. The strains were previously collected by Lomholt and Killian [12]. All strains were previously identified by $16 \mathrm{~S}$ rRNA amplicon sequencing, and, for the $P$. acnes isolates, further typed by multi-locus sequence typing (MLST) [12]. Detailed information about the origin and the phylotypes of the strains used in this study is given in Additional file 1 and Additional file 2.

Bacteria were cultivated on $5 \%$ sheep blood agar medium for one (S. epidermidis) or three ( $P$. acnes) days. S. epidermidis was cultivated at $37{ }^{\circ} \mathrm{C}$ aerobically, supplemented with $5 \% \mathrm{CO}_{2}$. P. acnes was cultivated at $37{ }^{\circ} \mathrm{C}$ in an anaerobic chamber, in an atmosphere of $80 \% \mathrm{~N}_{2}, 10 \% \mathrm{CO}_{2}$ and $10 \% \mathrm{H}_{2}$.

\section{Agar plate antagonism assay to screen for antimicrobial activity}

$P$. acnes strains were individually screened for antimicrobial activity against $S$. epidermidis indicator strains. The screening procedure was carried out as a simultaneous antagonism assay, as described by Tagg and Bannister, with slight modifications [58]. In brief, the screenings were carried out on large Trypticase Soy (TS) agar plates (14 $\mathrm{cm}$ diameter) by the following procedure: $150 \mu \mathrm{l}$ liquid TS broth cultures of individual $P$. acnes strains was aliquoted into wells of a flat-bottom 96-well microtiter plate. TS agar plates were floated with a lawn of the individual S. epidermidis (suspended in $0.85 \% \mathrm{NaCl}$ ) indicator strains. The concentrations of the indicator strain suspensions were standardized by comparison to a 0.5 McFarland standard $\left(150 \times 10^{6} \mathrm{CFU} / \mathrm{ml}, \mathrm{OD}=0.125\right)$. The plates were allowed to dry in a laminar flow bench for $15 \mathrm{~min}$. The $P$. acnes strains were then simultaneously 
point-inoculated with an in-house developed multiinoculator device onto the surface of the TS agar plates. The plates were incubated at $37^{\circ} \mathrm{C}$ for $72 \mathrm{~h}$, in an anaerobic chamber, in an atmosphere of $80 \% \mathrm{~N}_{2}, 10 \% \mathrm{CO}_{2}$ and $10 \% \mathrm{H}_{2}$, and examined for zones of growth inhibition.

The same approach was used to screen S. epidermidis strains for their antimicrobial activity against $P$. acnes indicator strains. TS agar plates were floated with a lawn of the individual $P$. acnes indicator strains. $20 \mathrm{~S}$. epidermidis strains were simultaneously point-inoculated onto the surface of the TS agar plates and the plates were incubated at $37^{\circ} \mathrm{C}$ for $72 \mathrm{~h}$ in an anaerobic chamber. In addition, plates were incubated in microaerophilic conditions, using the GasPak system (Oxoid, AnaeroJar) with CampyGen sachets. Each antagonism assay was carried out in triplicates.

\section{Building a S. epidermidis core genome phylogenetic tree} Genome sequences of $112 \mathrm{~S}$. epidermidis strains were used to build a phylogenetic tree based on all shared sequences (i.e., the core genome of the species). Genomes were downloaded from NCBI Genome and WGS databases, with the exception of strains AU21, AU23, AU24 and FS1 that were sequenced for this study. The core genome was identified by slicing the genome sequence of strain 14.1.R1 into fragments of $200 \mathrm{bp}$ which were used as query sequences in BLASTn (BLAST+) [59] to extract homologous sequences from the other 110 genomes. Default parameters of BLASTn were used together with a $65 \%$ cut-off on coverage. Homologous sequences of each fragment were aligned using Muscle [60] and subsequently all fragments were concatenated into one sequence per strain using a python script. The core genome phylogenetic tree was built in Mega v6 [61] using the minimal-evolution algorithm with pair-wise deletion and 100 bootstrap replications. A total of 139,881 single nucleotide polymorphisms were found.

\section{Genome sequencing}

Genomic DNA from S. epidermidis strains AU21, AU23, AU24 and FS1 was isolated using the MasterPure Grampositive DNA Purification Kit (EpiCentre MGP04100) according to the manufacturer's instructions. The purity and quality of the gDNA were assessed on a $1 \%$ agarose gel and with a nanodrop apparatus. For strains AU21, AU24 and FS1, library preparation and Illumina sequencing were carried out at the Göttingen Genomics Laboratory, Germany. Sequences were trimmed with Trimmomatic 0.32 (http://www.usadellab.org/cms/?page= trimmomatic) and assembled with SPAdes 3.1.1 (http:// bioinf.spbau.ru/en/spades), resulting in $2,421,814$ bp in 72 contigs (AU21), 2,429,243 bp in 61 contigs (AU24), and $2,551,451$ bp in 91 contigs (FS1), respectively. The GenBank accession numbers of the draft genome sequences are LNUN00000000 (AU21), LNSW00000000 (AU24) and LOAT00000000 (FS1).

The genome of $S$. epidermidis AU23 was sequenced on a PacBio RS I sequencing instrument using an $10 \mathrm{~kb}$ insert library (GATC Biotech AG, Germany), resulting in a total of 66,762 post-filtered continuous long reads with an average length of 3,687 bp. A sequence coverage of approximately 81 -fold was obtained after sequence read assembly, that resulted in a genome size of 2,487,973 bp split into 5 contigs. The GenBank accession number of the draft genome sequence of strain AU23 is LNUS00000000.

\section{Gene prediction, annotation and comparative genome analysis}

For S. epidermidis strains AU21, AU23, AU24, and FS1 open reading frames (ORFs) and tRNAs were identified and annotated using the RAST server [62]. Additional manual annotation of ORFs of interest was performed on the basis of homology searches with the public nonredundant $(\mathrm{nr})$ database using BLASTp. Domain search was done using PFAM (http://pfam.xfam.org/). For comparative genome analysis, the program BRIG was used [63]. The following genomes of $S$. epidermidis were taken from GenBank to build a BRIG genome comparison: 14.1.R1 [BioProject: PRJNA64683], VCU128 [BioProject: PRJNA53771], VCU118 [BioProject: PRJNA53755], VCU129 [BioProject: PRJNA53773], VCU071 [BioProject: PRJNA53733], VCU105 [BioProject: PRJNA53739], VCU 127 [BioProject: PRJNA53769], RP62A [BioProject: PRJ NA64], NIHLM023 [BioProject: PRJNA62371], NIH LM061 [BioProject: PRJNA62355], NIHLM067 [BioProject: PRJNA62353], FRI909 [BioProject: PRJNA48603], ATCC12228 [BioProject: PRJNA279].

\section{Partial purification of bioactive fraction from $S$. epidermidis 14.1.R1}

S. epidermidis strain 14.1.R1 was cultivated on 45 TS agar plates containing $0.1 \%(\mathrm{w} / \mathrm{v})$ skim milk at $37{ }^{\circ} \mathrm{C}$ in an aerobic atmosphere supplemented with $5 \% \mathrm{CO}_{2}$ for $24 \mathrm{~h}$. Bacteria were harvested from all plates and suspended in $225 \mathrm{ml} 0.9 \% \mathrm{NaCl}$ solution. The bacteria were then removed by centrifugation at $5000 \mathrm{~g}$ for $30 \mathrm{~min}$ at room temperature, followed by sterilization of the supernatant using $0.22 \mu \mathrm{m}$ filter disks (Sarstedt). The cell-free supernatant (CFS) was concentrated 75 -fold by centrifugal ultrafiltration on a Centriprep ${ }^{\oplus}$ centrifugal filter unit (Millipore) at $3000 \mathrm{~g}$ at $4{ }^{\circ} \mathrm{C}$. The protein content in the concentrated CFS (cCFS) was measured by UV absorption at $280 \mathrm{~nm}$. To assay for the potency of cCFS, multiple dilutions were made and tested for bioactivity against a sensitive $P$. acnes indicator strain by the spot-onlawn assay; a volume of $10 \mu \mathrm{l}$ cCFS was spotted onto the 
centre of a pre-dried $5 \%$ sheep blood agar plate seeded with a suspension of a $P$. acnes indicator strain $(0.5$ McFarland standard: $150 \times 10^{6} \mathrm{CFU} / \mathrm{ml}, \mathrm{OD}_{550 \mathrm{~nm}}=$ 0.125). The plates were incubated at $37^{\circ} \mathrm{C}$ in an anaerobic chamber, in an atmosphere of $80 \% \mathrm{~N}_{2}, 10 \% \mathrm{CO}_{2}$ and $10 \% \mathrm{H}_{2}$, for $48 \mathrm{~h}$. A clear zone of growth inhibition surrounding the spotted samples indicated antimicrobial activity.

Effect of proteinase $\mathrm{K}, \mathrm{pH}$, heat treatment and storage on antimicrobial activity

The sensitivity of the cCFS to proteolytic activity was tested, employing a $2 \mathrm{~h}$ treatment with proteinase $\mathrm{K}$ at $37^{\circ} \mathrm{C}$ at a final concentration of $10 \mathrm{mg} / \mathrm{ml}$. After incubation, the cCFS was assayed for residual activity. In order to determine the effect on $\mathrm{pH}$ on bioactivity, the $\mathrm{pH}$ of the cCFS was adjusted to $\mathrm{pH} 2$ using $1 \mathrm{M} \mathrm{HCl}$ and incubated at RT for $2 \mathrm{~h}$, before neutralizing. The cCFS was subsequently assayed for residual activity. To determine the thermal stability, the cCFS was heated at 60 and $80{ }^{\circ} \mathrm{C}$ for 10,30 or $60 \mathrm{~min}$, respectively, after which each sample was assayed for residual activity. The cCFS was stored at $-20{ }^{\circ} \mathrm{C}$ and $4{ }^{\circ} \mathrm{C}$. At different time intervals, between $24 \mathrm{~h}$ and 120 days, the cCFS was assayed for residual activity. Containers used in the following experiments were coated with Emulphogene (Polyoxyethylene 10 Tridecyl Ether, Sigma-Aldrich) in order to prevent binding of the active substance to the container surface.

\section{Gel electrophoresis and mass spectrometry}

The cCFS was subjected to protein identification. Since most antimicrobial substances are low molecular weight (LMW) compounds we focussed on the analysis of LMW proteins, using tricine-sodium dodecyl sulphatepolyacrylamide gel electrophoresis (tricine-SDS-PAGE) [64]. Polyacrylamide concentration in the separating gel was $18 \%$. Electrophoresis was conducted at a constant voltage of $50 \mathrm{~V}$ for $30 \mathrm{~min}$, followed by $120 \mathrm{~V}$ for $4 \mathrm{~h}$. A polypeptide SDS-PAGE molecular weight standard (BioRad) with sizes ranging from 1.06 to $26.6 \mathrm{kDa}$ was included. After electrophoresis the gel was silver stained and bands were selected for Matrix-Assisted Laser Desorption/Ionization Quadrupole Time-Of-Flight (MALDIQ-TOF) Mass Spectrometry (MS). Individual protein bands were excised from the gel, destained and subjected to in-gel tryptic digestion as described previously [65]. The extracted peptides were desalted using $\mathrm{C}_{18}$ Stage Tips (Proxeon, Thermo Scientific) and the adsorbed peptides were eluted directly onto a Bruker Autoflex MSP 96 Stainless Steel Target Plate using $1 \mu$ l MALDI-matrix solution (2 $\mathrm{mg} / \mathrm{mL}$-cyano-4-hydroxy-cinnamic acid (CHCA),

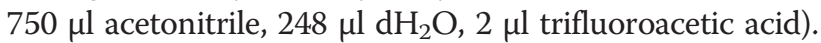
The solvent was allowed to evaporate leaving matrix crystals in which the tryptic peptides were embedded. The mass-to-charge $(\mathrm{m} / \mathrm{z})$ ratios of eluted peptides were analysed on a MALDI-Q-TOF Mass Spectrometer (Micromass Q-Tof Ultima Global, Walters). Fibrinopeptide B (MW: $1570.6 \mathrm{Da}$ ) was used as an internal standard for all obtained MS data. All generated MS and MS/MS data were processed using the Mascot software [66] (www.matrixscience.com). For peptide mass fingerprinting (PMF), mass spectra were acquired in the positive-ion mode over the range $700-3500 \mathrm{~m} / z$. The search parameters allowed for variable modifications of methionine oxidation and fixed modifications of cysteine propionamide adduct. There was a peptide mass tolerance of $1.2 \mathrm{Da}$, and a single missed tryptic cleavage was allowed in the search. The generated MS/MS data were examined using the MS/MS Ion Search option in Mascot, with a fragment mass tolerance of $0.6 \mathrm{Da}$, and allowance of 1 missed cleavage. Proteins were identified via database queries with the search engine Mascot in three databases (NCBI-nr, Swiss-Prot and Mass Spectrometry Protein Sequence Database (MSDB)). The MS data were also searched directly against the proteome of S. epidermidis 14.1.R1. The probability score calculated by the software was used as the criterion for correct identification [66].

\section{Ethics approval and consent to participate}

The study protocol was approved by the Ethics Committee of the County of Aarhus, and the study was conducted according to the principles of the declaration of Helsinki. Written informed consent was obtained from study participants and/or their legal guardians.

\section{Availability of data and materials}

The Whole Genome Shotgun projects of S. epidermidis strains have been deposited at DDBJ/EMBL/GenBank under the accession numbers: LNUN00000000 (AU21), LNUS00000000 (AU23), LNSW00000000 (AU24) and LOAT00000000 (FS1). Other supporting data are included as Additional files.

\section{Additional files}

Additional file 1: $P$. acnes strains used in this study. (DOCX $35 \mathrm{~kb}$ ) Additional file 2: $S$. epidermidis strains used in this study. (DOCX $17 \mathrm{~kb}$ )

Additional file 3: Inhibitory activity of 77 P. acnes strains against 12 S. epidermidis strains. (DOCX $31 \mathrm{~kb})$

Additional file 4: Sensitivity of individual $P$. acnes strains against the antimicrobial activity of $20 \mathrm{~S}$. epidermidis strains. (DOCX $16 \mathrm{~kb}$ ) Additional file 5: Antimicrobial activity spectrum of S. epidermidis strain 14.1.R1. (DOCX $19 \mathrm{~kb})$

Additional file 6: A. Genome comparison of 17 S. epidermidis strains with strain AU23 as reference. Newly sequenced genomes were included (FS1, AU21, AU24). The other 13 genomes were selected 
based on their position in the phylogenetic core genome tree (Fig. 2). Regions of genomic variability (1-7) can be detected (see Additional file $7 \mathrm{~A}$ for their gene content). The BRIG program was used to generate the figure. B. Genome comparison of $17 \mathrm{~S}$. epidermidis strains with strain FS1 as reference. Newly sequenced genomes were included (AU21, AU23, AU24). The other 13 genomes were selected based on their position in the phylogenetic core genome tree (Fig. 2). Regions of genomic variability can be detected (see Additional file 7B for their gene content). The BRIG program was used to generate the figure. (DOCX $1185 \mathrm{~kb}$ )

Additional file 7: A: Seven genomic islands in strain S. epidermidis AU23. B: Ten genomic islands in strain S. epidermidis FS1. C: Eight genomic islands in strain S. epidermidis 14.1.R1. (XLSX $331 \mathrm{~kb}$ )

\section{Additional file 8: SDS-PAGE analysis of concentrated cell-free} supernatant (CCFS) of S. epidermidis 14.1.R1. Different concentrations of the S. epidermidis 14.1.R1 CCFS separated on an $18 \%$ polyacrylamide gel and stained with the silver stain method. Lane 1: broad range molecular weight standard. Lanes 2-6: different concentrations of S. epidermidis 14.1.R1 CCFS. The gel bands that were analyzed with MALDI-TOF MS are marked A-C. Proteins were identified using Mascot Search of MS/MS data from the peptide digest with databases NCBI-nr, MSDB, and Swiss-Prot (and using the 14.1.R1 genome): A, HMPREF9956_2246 (EsXA) (MW: 11015 Da); B, HMPREF9956_1196 (DNA-binding protein HU (MW: 9626 Da): C, HMPREF9956_0860 and HMPREF9956_0861 (PSMB1a/1b) (MW: 4639 Da)/HMPREF9956_0859 (PSMR2) (MW: 4644 Da). (DOCX 368 kb)

\section{Additional file 9: A. Amino acid sequences of the PSM $\beta$-proteins} from S. epidermidis 14.1.R1. The sequence shown in bold red represents the sequence coverage by MS analysis. The sequence coverage was $70 \%$ and $40 \%$ for PSM 32 and PSM $1 \mathrm{a} / 1 \mathrm{~b}$, respectively. B. The $p s m \beta$ operon is composed of four genes in the S. epidermidis strain 14.1.R1. The two peptides PSMB1a (HMPREF9956_0861), and PSMB1b (HMPREF9956_0860) are identical on protein level; on DNA level there are 3 single nucleotide polymorphisms. (DOCX $26 \mathrm{~kb}$ )

Additional file 10: Amino acid sequence alignment of EsxA from S. epidermidis 14.1.R1, S. aureus M0733 and M. tuberculosis H37Rv. The Trp-Xaa-Gly (WXG) motif, a signature sequence of ESAT-6-like proteins, is marked with three asterisks. The EsXA protein is structurally organized as a helical hairpin, with the conserved WXG motif that localizes in a loop between the two a-helices. (DOCX $82 \mathrm{~kb}$ )

\section{Abbreviations}

ADI: arginine deiminase; cCFS: concentrated cell-free supernatant; CDI: contact-dependent growth inhibition; ESAT-6: early secretory antigenic 6 kDa; ESS: ESAT-6 secretion system; MGE: mobile genetic element; SaPI: S. aureus pathogenicity island.

\section{Competing interests}

The authors declare that they have no competing interests.

\section{Authors' contributions}

HB, HBL and MK conceptualized and designed the study. GJMC performed antagonism assays and CCFS preparations. Proteomics work was done by JJ and GJMC. CFPS carried out phylogenetic analyses. Genome sequencing was done by AT and EB. HB analysed genome data. HR provided materials and advice. GJMC and HB wrote the manuscript and all authors read and approved the final manuscript.

\section{Acknowledgements}

The authors want to thank Estelle Marchal and Herdis Johansen for excellent technical assistance and Andreas Leimbach for bioinformatics advice.

\section{Funding}

This work was supported by the Danish Medical Research council (DFF-1331-00241 to HB) (http://ufm.dk). The funders had no role in study design, data collection and analysis, decision to publish, or preparation of the manuscript.

\section{Author details}

'Department of Biomedicine, Aarhus University, Aarhus, Denmark

2Department of Molecular Biology and Genetics, Aarhus University, Aarhus,
Denmark. ${ }^{3}$ Institute of Medical Microbiology, University Hospital Hamburg-Eppendorf, Hamburg, Germany. ${ }^{4}$ Department of Genomic and Applied Microbiology, Institute of Microbiology and Genetics, Georg-August University Göttingen, Göttingen, Germany.

Received: 10 December 2015 Accepted: 17 February 2016

Published online: 29 February 2016

\section{References}

1. Findley K, Oh J, Yang J, Conlan S, Deming C, Meyer JA, et al. Topographic diversity of fungal and bacterial communities in human skin. Nature. 2013; 498:367-70.

2. Oh J, Byrd AL, Deming C, Conlan S, Kong HH, Segre JA. Biogeography and individuality shape function in the human skin metagenome. Nature. 2014; 514:59-64.

3. Grice EA, Segre JA. The skin microbiome. Nat Rev Microbiol. 2011;9:244-53.

4. Vuong C, Otto M. Staphylococcus epidermidis infections. Microbes Infect. 2002;4:481-9.

5. Coates R, Moran J, Horsburgh MJ. Staphylococci: colonizers and pathogens of human skin. Future Microbiol. 2014:9:75-91.

6. Christensen GJ, Brüggemann H. Bacterial skin commensals and their role as host quardians. Benef Microbes. 2014:5:201-15.

7. Perry A, Lambert P. Propionibacterium acnes: infection beyond the skin. Expert Rev Anti Infect Ther. 2011:9:1149-56.

8. McDowell A, Patrick S, Eishi Y, Lambert P, Eady A. Propionibacterium acnes in human health and disease. Biomed Res Int. 2013;2013:493564.

9. van Rensburg JJ, Lin H, Gao X, Toh E, Fortney KR, Ellinger S, Zwickl B, Janowicz DM, Katz BP, Nelson DE, Dong Q, Spinola SM. The human skin microbiome associates with the outcome of and is influenced by bacterial infection. mBio. 2015;6(5):e01315-15. doi:10.1128/mBio.01315-15.

10. Williams HC, Dellavalle RP, Garner S. Acne vulgaris. Lancet. 2012;379:361-72.

11. Bek-Thomsen M, Lomholt HB, Kilian M. Acne is not associated with yet-uncultured bacteria. J Clin Microbiol. 2008;46:3355-60.

12. Lomholt HB, Kilian M. Population genetic analysis of Propionibacterium acnes identifies a subpopulation and epidemic clones associated with acne. PLoS One. 2010;5:e12277.

13. Conlan S, Mijares LA, NISC Comparative Sequencing Program, Becker J, Blakesley RW, Bouffard GG, et al. Staphylococcus epidermidis pan-genome sequence analysis reveals diversity of skin commensal and hospital infection-associated isolates. Genome Biol. 2012;13:R64.

14. McDowell A, Valanne S, Ramage G, Tunney MM, Glenn JV, McLorinan GC, et al. Propionibacterium acnes types I and II represent phylogenetically distinct groups. J Clin Microbiol. 2005:43:326-34.

15. McDowell A, Gao A, Barnard E, Fink C, Murray PI, Dowson CG, et al. A novel multilocus sequence typing scheme for the opportunistic pathogen Propionibacterium acnes and characterization of type I cell surfaceassociated antigens. Microbiology. 2011;157:1990-2003.

16. McDowell A, Barnard E, Nagy I, Gao A, Tomida S, Li H, et al. An expanded multilocus sequence typing scheme for Propionibacterium acnes: investigation of 'pathogenic', 'commensal' and antibiotic resistant strains. PLoS One. 2012;7:e41480

17. Fitz-Gibbon S, Tomida S, Chiu BH, Nguyen L, Du C, Liu M, et al. Propionibacterium acnes strain populations in the human skin microbiome associated with acne. J Invest Dermatol. 2013;133:2152-60.

18. Brzuszkiewicz E, Weiner J, Wollherr A, Thürmer A, Hüpeden J, Lomholt HB, et al. Comparative genomics and transcriptomics of Propionibacterium acnes. PLoS One. 2011;6:e21581.

19. Brüggemann $H$, Lomholt HB, Kilian M. The flexible gene pool of Propionibacterium acnes. Mob Genet Elements. 2012;2:145-8.

20. Chen HJ, Chang YC, Tsai JC, Hung WC, Lin YT, You SJ, et al. New structure of phage-related islands carrying fusB and a virulence gene in fusidic acid-resistant Staphylococcus epidermidis. Antimicrob Agents Chemother. 2013:57:5737-9.

21. Marquis RE, Bender GR, Murray DR, Wong A. Arginine deiminase system and bacterial adaptation to acid environments. Appl Environ Microbiol. 1987;53:198-200.

22. Speziale P, Pietrocola G, Foster TJ, Geoghegan JA. Protein-based biofilm matrices in Staphylococci. Front Cell Infect Microbiol. 2014;4:171.

23. Wang Y, Kuo S, Shu M, Yu J, Huang S, Dai A, et al. Staphylococcus epidermidis in the human skin microbiome mediates fermentation to inhibit 
the growth of Propionibacterium acnes: implications of probiotics in acne vulgaris. Appl Microbiol Biotechnol. 2014;98:411-24.

24. Novick RP, Christie GE, Penadés JR. The phage-related chromosomal islands of Gram-positive bacteria. Nat Rev Microbiol. 2010;8:541-51.

25. Heilmann C, Schweitzer O, Gerke C, Vanittanakom N, Mack D, Götz F. Molecular basis of intercellular adhesion in the biofilm-forming Staphylococcus epidermidis. Mol Microbiol. 1996;20:1083-91.

26. Schnell N, Entian KD, Schneider U, Götz F, Zähner H, Kellner R, et al. Prepeptide sequence of epidermin, a ribosomally synthesized antibiotic with four sulphide-rings. Nature. 1988;333:276-8.

27. Geissler S, Götz F, Kupke T. Serine protease EpiP from Staphylococcus epidermidis catalyzes the processing of the epidermin precursor peptide. J Bacteriol. 1996;178:284-8.

28. Rigel NW, Braunstein M. A new twist on an old pathway-accessory Sec systems. Mol Microbiol. 2008;69:291-302.

29. Tormo MA, Knecht E, Götz F, Lasa I, Penadés JR. Bap-dependent biofilm formation by pathogenic species of Staphylococcus: evidence of horizontal gene transfer? Microbiology. 2005;151:2465-75.

30. Abdallah AM, van Pittius NC G, Champion PA, Cox J, Luirink J, VandenbrouckeGrauls CM, et al. Type VII secretion-mycobacteria show the way. Nat Rev Microbiol. 2007;5:883-91.

31. Burts ML, Williams WA, DeBord K, Missiakas DM. EsxA and EsxB are secreted by an ESAT-6-like system that is required for the pathogenesis of Staphylococcus aureus infections. Proc Natl Acad Sci U S A. 2005;102: 1169-74.

32. Burts ML, DeDent AC, Missiakas DM. EsaC substrate for the ESAT-6 secretion pathway and its role in persistent infections of Staphylococcus aureus. Mol Microbiol. 2008;69:736-46.

33. Peschel A, Otto M. Phenol-soluble modulins and staphylococcal infection. Nat Rev Microbiol. 2013;11:667-73.

34. Wang R, Khan BA, Cheung GY, Bach TH, Jameson-Lee M, Kong KF, et al. Staphylococcus epidermidis surfactant peptides promote biofilm maturation and dissemination of biofilm-associated infection in mice. J Clin Invest. 2011;121:238-48.

35. Mehlin C, Headley CM, Klebanoff SJ. An inflammatory polypeptide complex from Staphylococcus epidermidis: isolation and characterization. J Exp Med. 1999;189:907-18.

36. Holland KT, Cunliffe WJ, Eady EA. Intergeneric and intrageneric inhibition between strains of Propionibacterium acnes and micrococcaceae, particularly Staphylococcus epidermidis, isolated from normal skin and acne lesions. J Med Microbiol. 1979;12:71-82.

37. Malcolmson SJ, Young TS, Ruby JG, Skewes-Cox P, Walsh CT. The posttranslational modification cascade to the thiopeptide berninamycin generates linear forms and altered macrocyclic scaffolds. Proc Natl Acad Sci U S A. 2013;110:8483-8.

38. Walsh $C T$, Acker MG, Bowers AA. Thiazolyl peptide antibiotic biosynthesis: a cascade of post-translational modifications on ribosomal nascent proteins. J Biol Chem. 2010;285:27525-31.

39. Just-Baringo $X$, Albericio F, Álvarez M. Thiopeptide antibiotics: retrospective and recent advances. Mar Drugs. 2014;12:317-51.

40. Schoenfelder SM, Lange C, Eckart M, Hennig S, Kozytska S, Ziebuhr W. Success through diversity - how Staphylococcus epidermidis establishes as a nosocomial pathogen. Int J Med Microbiol. 2010;300:380-6.

41. Lindsay JA. Staphylococcus aureus genomics and the impact of horizontal gene transfer. Int J Med Microbiol. 2014;304:103-9.

42. Alibayov B, Baba-Moussa L, Sina H, Zdeňková K, Demnerová K. Staphylococcus aureus mobile genetic elements. Mol Biol Rep. 2014;41: 5005-18.

43. Lindgren JK, Thomas VC, Olson ME, Chaudhari SS, Nuxoll AS, Schaeffer CR, et al. Arginine deiminase in Staphylococcus epidermidis functions to augment biofilm maturation through pH homeostasis. J Bacteriol. 2014; 196:2277-789.

44. Manosroi A, Khanrin P, Lohcharoenkal W, Werner RG, Götz F, Manosroi W, et al. Transdermal absorption enhancement through rat skin of gallidermin loaded in niosomes. Int J Pharm. 2010;392:304-10.

45. Gey Van Pittius NC, Gamieldien J, Hide W, Brown GD, Siezen RJ, Beyers AD. The ESAT-6 gene cluster of Mycobacterium tuberculosis and other high G+C Gram-positive bacteria. Genome Biol. 2001;2:Research0044.

46. Berthet FX, Rasmussen PB, Rosenkrands I, Andersen P, Gicquel B. A Mycobacterium tuberculosis operon encoding ESAT-6 and a novel low-molecular-mass culture filtrate protein (CFP-10). Microbiology. 1998;144:3195-203.

47. Stanley SA, Raghavan S, Hwang WW, Cox JS. Acute infection and macrophage subversion by Mycobacterium tuberculosis require a specialized secretion system. Proc Natl Acad Sci U S A. 2003;100:13001-6.

48. Davis JM, Ramakrishnan L. The role of the granuloma in expansion and dissemination of early tuberculous infection. Cell. 2009;136:37-49.

49. Renshaw PS, Lightbody KL, Veverka V, Muskett FW, Kelly G, Frenkiel TA, et al. Structure and function of the complex formed by the tuberculosis virulence factors CFP-10 and ESAT-6. Embo J. 2005;24:2491-8.

50. Pallen MJ. The ESAT-6/WXG100 superfamily - and a new Gram-positive secretion system? Trends Microbiol. 2002;10:209-12.

51. Sundaramoorthy R, Fyfe PK, Hunter WN. Structure of Staphylococcus aureus EsXA suggests a contribution to virulence by action as a transport chaperone and/or adaptor protein. J Mol Biol. 2008;383:603-14.

52. Korea CG, Balsamo G, Pezzicoli A, Merakou C, Tavarini S, Bagnoli F, et al. Staphylococcal Esx proteins modulate apoptosis and release of intracellular Staphylococcus aureus during infection in epithelial cells. Infect Immun. 2014:82:4144-53.

53. Zhang $D$, lyer $L M$, Aravind L. A novel immunity system for bacterial nucleic acid degrading toxins and its recruitment in various eukaryotic and DNA viral systems. Nucleic Acids Res. 2011;39:4532-52.

54. Zhang D, de Souza RF, Anantharaman V, lyer LM, Aravind L. Polymorphic toxin systems: Comprehensive characterization of trafficking modes, processing, mechanisms of action, immunity and ecology using comparative genomics. Biol Direct. 2012;7:18.

55. Jamet A, Nassif X. New players in the toxin field: polymorphic toxin systems in bacteria. mBio. 2015;6(3):e00285-15. doi:10.1128/mBio.00285-15.

56. Aoki SK, Diner EJ, de Roodenbeke CT, Burgess BR, Poole SJ, Braaten BA, et al A widespread family of polymorphic contact-dependent toxin delivery systems in bacteria. Nature. 2010;468:439-42.

57. Aoki SK, Pamma R, Hernday AD, Bickham JE, Braaten BA, Low DA. Contactdependent inhibition of growth in Escherichia coli. Science. 2005;309:1245-8.

58. Tagg JR, Bannister LV. "Fingerprinting" beta-haemolytic streptococci by their production of and sensitivity to bacteriocine-like inhibitors. J Med Microbiol. 1979;12:397-411.

59. Camacho C, Coulouris G, Avagyan V, Ma N, Papadopoulos J, Bealer K, et al. BLAST+: architecture and applications. BMC Bioinformatics. 2009;10:421.

60. Edgar RC. MUSCLE: multiple sequence alignment with high accuracy and high throughput. Nucleic Acids Res. 2004;32:1792-7.

61. Tamura K, Stecher G, Peterson D, Filipski A, Kumar S. MEGA6: Molecular Evolutionary Genetics Analysis version 6.0. Mol Biol Evol. 2013;30:2725-9.

62. Aziz RK, Bartels D, Best AA, DeJongh M, Disz T, Edwards RA, et al. The RAST Server: rapid annotations using subsystems technology. BMC Genomics. 2008;9:75.

63. Alikhan NF, Petty NK, Ben Zakour NL, Beatson SA. BLAST Ring Image Generator (BRIG): simple prokaryote genome comparisons. BMC Genomics. 2011;12:402.

64. Schagger $\mathrm{H}$, von Jagow G. Tricine-sodium dodecyl sulfate-polyacrylamide gel electrophoresis for the separation of proteins in the range from 1 to 100 kDa. Anal Biochem. 1987;166:368-79.

65. Shevchenko A, Wilm M, Vorm O, Mann M. Mass spectrometric sequencing of proteins silver-stained polyacrylamide gels. Anal Chem. 1996;68:850-8.

66. Perkins DN, Pappin DJ, Creasy DM, Cottrell JS. Probability-based protein identification by searching sequence databases using mass spectrometry data. Electrophoresis. 1999;20:3551-67.

\section{Submit your next manuscript to BioMed Central and we will help you at every step:}

- We accept pre-submission inquiries

- Our selector tool helps you to find the most relevant journal

- We provide round the clock customer support

- Convenient online submission

- Thorough peer review

- Inclusion in PubMed and all major indexing services

- Maximum visibility for your research

Submit your manuscript at www.biomedcentral.com/submit 